\title{
THE INFLUENCE OF RANKISM ON THE ETHICAL COMPETENCE OF STUDENT NURSES IN A MILITARY CONTEXT
}

\section{Karen Zägenhagen and Gisela van Rensburg University of South Africa}

\begin{abstract}
Developing ethical competence in nursing is integral to the process of professional socialisation during which students are taught the ethical values and norms of the nursing profession. The process of professional socialisation is however influenced by a range of factors related to the teaching and learning environment, such as organisational culture, teaching practices and role models. Students at a military nursing college are exposed to a unique environment rich in military culture. The findings discussed in this article formed part of a larger grounded theory study that aimed to determine the influence of the hidden curriculum on the professional socialisation of students in a military context. Data were collected from purposefully selected nurse educators and students by means of focus-group interviews and critical incident narratives. Findings revealed a significant influence of the abuse of rank on the ethical competence of students.
\end{abstract}

Keywords: ethical competence, hidden curriculum, military teaching and learning environment, professional socialisation, rankism

\section{Introduction}

Nursing is a science and a profession founded on specific human values emphasising the importance of ethical values. ${ }^{1}$ The professional values associated with nursing serve to guide nurses' behaviour to comply with the preferred standards of the profession. This forms the foundation for the provision of committed, caring, safe and ethical healthcare. Values have been described as basic convictions of what is right, good or desirable, and influence both social and professional behaviour. ${ }^{2}$

The development of professional values and value-based behaviours, which is crucial in ensuring improved quality of nursing care, is described as professional socialisation. ${ }^{3}$ Professional socialisation involves inter alia the development of students' ethical competence. 
Numerous studies have established that most of what is learned during the process of professional socialisation takes place not within the formal course curriculum, but by means of interactions and influences that are intrinsic to the teaching and learning environment. ${ }^{4,5}, 6$ These perspectives on the teaching of professionalism and the process of professional socialisation within a multidimensional teaching and learning environment suggest the existence of a hidden curriculum.

Hafferty and O'Donnell define the hidden curriculum as the attitudes and values conveyed in an implicit and tacit fashion, often unintentionally, through the educational structures, practices and culture of an educational institution. ${ }^{7}$ In addition, Numminen, Leino-Kilpi, Isoaho and Meretoja state that the social framework in which environmental elements and interpersonal relations interact, are affecting nurses' professional and ethical practice. ${ }^{8}$

In order to train professional nurses who possess the necessary professional values, the goal of nursing education should be to develop students' ethical competence. From the abovementioned, it can however be deduced that the teaching and learning context, environment and interactions, play a significant role in the development of ethical competence.

Nursing education institutions (NEIs) are multidimensional organisations with complex relations and dynamics. Hafferty and O'Donnell claim that the more complex an organisational setting, the more likely it is that there will be disjuncture between what is being taught and what is being learnt. ${ }^{9}$

A military nursing college, where students are trained to be competent professional military nurses, is no less complex. Due to the uniqueness of the military environment, the professional socialisation of student nurses is compounded by simultaneous military socialisation within a teaching and learning environment inundated with military culture. ${ }^{10}$

At this point, it is important to distinguish between rank and rankism. The former describes a position in an organisation, such as the military, to indicate the seniority of the bearer. ${ }^{11}$ Rankism, on the other hand, is the abuse of this position. It includes the assertion of superiority and the discrimination against people on the grounds of rank. ${ }^{12}$

The highly authoritarian structure of the military clearly delineates ranks and responsibilities. It further ensures an organisational boundary system to regulate and maintain professional and personal relationships. Staal and King describe how power imbalances in the military are evident from the emphasis on rank. ${ }^{13}$ The students in this study on the nature of the hidden curriculum in a military teaching and learning environment, were candidate officers (COs), a temporary rank given to them until they have successfully completed their studies, after which they are appointed as officers. The rank of CO is thus subordinate to all other ranks.

The adherence of the military to hierarchy relates to its "acceptance of implicit and explicit authority distinctions in professional and social relationships". ${ }^{14}$ In the 
military, members in authority positions direct and control the organisation, and their leadership and authority are based on formal status and seniority that should be clearly visible. People who are not part of the military organisation, may see the display of authority, channels of command and ranks as trivial, but Jans nevertheless alerts us to the fact that there is far more to authority than that. ${ }^{15}$ Command positions and rank insignia denote professional obligations that go beyond leadership and the control of subordinates. Military command is as much a system of mutual service and selfrespect as it is one of perceived domination, compliance and subservience. The nature of the military organisation, although bureaucratic, is also very autocratic. Support and loyalty of subordinates are not credited to the person but rather to his or her position. Military hierarchy lends its strength to its role in times of crisis, danger and uncertainty, conditions that are, after all, the reason why the military exists. ${ }^{16}$

Despite the rationale for military ranks within the military hierarchical structure, military nursing colleges still have a moral obligation to train ethically competent nurses. Cannaerts, Gastmans and De Casterle define ethical competence as the possession of ethical knowledge or ethical perception (the ability to 'see' what a situation presents), ethical reflection (to reflect critically about what nurses know, are and do) and ethical behaviour (to bring out the ethical practice and to 'be' ethical). ${ }^{17}$

An ethical competent student will display moral courage. This implies the willingness to stand up for and act according to his or her ethical beliefs when moral principles are threatened, regardless of the perceived or actual risks, such as stress, anxiety, isolation from colleagues, or threats to employment. In the absence of ethical competence, the student will feel morally distressed or powerless to act when witnessing improper behaviour as a result of organisational constraints that make doing the right thing difficult or impossible. ${ }^{18}$

Although authors like Fuller, Clarke and Bleich have written about rankism in nursing and healthcare and Murphy, Zangaro and Gadsden published a study on students' experiences with military nurses as educators, the researcher was unable to find any literature related to the effect of military rankism on the development of ethical competence of students. ${ }^{19}$

Given the unique teaching and learning environment in which military students find themselves, it is difficult to determine whether the military nursing education environment, where ranks mostly dictate interaction, nourishes the desirable professional attributes of a military nurse. The question that arose was whether it is likely that rankism in the military teaching and learning environment influences the development of students' ethical competence.

The purpose of this article is therefore to report on the significant influence of rankism on the ethical competence of students in a military context. 


\section{Methodology}

This article is based on the findings of a larger constructivist grounded theory study, which aimed to explore the nature of the hidden curriculum in a military teaching and learning environment, and to determine its influence on students' professional socialisation.

The research setting comprised a military nursing college, the selected military hospital and various governmental health facilities at which students are placed for clinical experience.

The researcher employed a non-probability sampling approach to select the study participants from nurse educators and students of a military nursing college. The nurse educators were selected by means of purposive sampling. The nurse educators had to be registered nurses with at least three years' experience as an educator at the military nursing college in order to be eligible for participation in the study. The sample from the students was obtained through convenience sampling. The students had to be registered for the four-year Diploma in Nursing (General, Community and Psychiatry) and Midwifery, and had to be in the fourth year of training for eligibility to participate in the study. Of the 23 nurse educators who met the criteria and were invited, 15 consented to participate, and from the entire group of 56 students who were invited participate, 12 consented.

The study was conducted in three phases. During Phase 1, the researcher employed focus-group interviews and critical incident narratives to address the research objectives.

The researcher applied Charmaz's approach to analyse the data collected during Phase $1 .^{20}$ This data analysis process included open coding, focused coding, theoretical sorting, diagramming, theoretical integration and theoretical coding. The researcher further used theoretical sensitivity, intuiting, memo-ing, reflexivity, constant comparison and theoretical sampling to ensure data quality.

The data-collection process resulted in two separate data sets. The first data set comprised the focus-group interviews with nurse educators. The second data set included both focus-group interviews with students and their critical incident narratives. During the second phase of the study, the findings of these two data sets were integrated. Phase 3 of the study set out to address the third objective of the study, namely to develop a substantive model, but this is beyond the scope of this article.

The researcher considered the three primary ethical principles on which research must be based, namely beneficence, respect for human dignity and justice as articulated in the Belmont Report. ${ }^{21}$ Ethical clearance was obtained from the academic institution where the study was conducted and the Military Hospital Research Ethics Committee. In order to ensure institutional protection, the researcher obtained permission from the Nursing College and Defence Intelligence to conduct the study. 


\section{Findings}

The integrated findings yielded four themes and 17 categories. The four themes related to the processes and experiences of nurse educators and students in the uniquely military contextual environment, namely:

- Theme 1: You're in the army now! - military acculturation

- Theme 2: Off to boot camp - professional knowledge acquisition

- Theme 3: Off to the battlefield - clinical skills acquisition

- Theme 4: Fighting a dichotomy - professional role conflict

Theme 1, "You're in the army now", represents the process of military acculturation as a result of military training. The metaphor was deemed appropriate in that the categories that emerged from the data encompassed aspects related to the participants' transition from civilians to soldiers. During this process, the student is introduced to military life and military culture, practices and values. ${ }^{22}$ Successful military acculturation leads to military socialisation.

The process of professional knowledge acquisition, represented by Theme 2, "Off to boot camp" refers to the process where the theory of nursing is taught and learned. Professional knowledge acquisition emerged as a dominant theme because of numerous references made to the adverse conditions in the military environment; the fact that the military environment is regarded as regulated, structured, restrictive, punitive and prejudiced; and the interference of military activities on nursing training.

The process of clinical skills acquisition is represented by Theme 3, "Off to the battlefield". Students are placed in a variety of healthcare facilities in order to gain clinical exposure and utilise clinical learning opportunities. The experiences of students in the clinical learning environment as reflected in the findings of this study confirm the critical influence of this process on their professional socialisation.

Theme 4, "Fighting a dichotomy" refers to the professional role conflict experienced by students in terms of being a soldier as well as a nurse. This duality explains the countless contradictions and conflicting messages experienced by students throughout the processes of military acculturation, professional knowledge acquisition and clinical skills acquisition, and attributed to most of the identified hidden elements in this study.

Although the integrated findings represent the experiences of both nurse educators and students, the findings reflected in this article, were purposefully extracted from the data set containing the experiences of the students. Numerous references by the students regarding the hierarchical environment, power abuse, exploitation of power, the autocratic approach, power and authority, and the abuse and exploitation of rank led to formulation of rankism as a category. Ethical issues related to rankism were also evident in all the themes. 
The hierarchical organisational structure of the military has been formalised by the use of military ranks. Military ranking and military customs and etiquette go hand in hand. The issue of rank is however considered to be a factor that contributes to the fact that students are reluctant to ask questions and moreover creates confusion in terms of ethical decision-making. As a result, students are often confronted with choosing between doing what they know is right and doing what they are instructed to do. Participants also debated the ethical conflict that students often experience when confronted with a military order or instruction from a higher-ranking officer, one which may be in contravention of the patient's right to privacy and confidentially. All direct quotations below are reproduced verbatim and unedited.

[T] he issue of ranks also come into play when you are taught to respect a higher ranking. When higher-ranking officers come into the ward, you have been taught to respect this person. So here comes this general, he is asking me ... about the private's condition in the ward. Do I now ...? We have spoken about the patient's right. Do I respect the patient's right to privacy and confidentiality or do I respect the higher-ranking officer who is asking me to divulge the person's private information and give [the information]? (Participant FGL1-3)

Stemming from the above, the ranking system is deemed one of the main challenges with regard to ethical practice. The participants acknowledged that nurse educators have a role to play in teaching students that patients have to be treated according to their needs and not their rank.

Another problem is that you as a nurse, you are trained to prioritise to triage in the ... like for instance in Casualty. The problem is that in the Defence Force when this general comes in, whether he comes in walking and looks healthy and all that, just with a headache, then the general has to be seen first before this critical patient that needs to be seen by the health professionals. (Participant FGL1-2)

I mean, whether you are a general, patients are treated according to priority. So, I mean, that is how you teach our students. Whether the patient comes in and is a general's wife that is pregnant and the other wife has got a blood pressure of $180 / 100$ whether it is a corporal, who do you give attention to first? Obviously, the patient that is more ill. But that again depends on how we teach our students. For me, it is about being an example, and then seeing the decision-making done by you. For me, it is really about seeing what we do, because that is mostly how students learn, by looking at what we do. (Participant FGL2-2) 
In the military profession, a higher-ranking member will always take precedence over one with a lower rank, and if a soldier fails to respect this, he or she will be regarded as disrespectful and ill-disciplined. In the nursing profession, however, patients are prioritised according to their health needs. If a nurse fails to treat patients based on their needs, it will be regarded as professional or ethical misconduct. Participants claim that military expectations often outweigh professional expectations because nurses are not sufficiently assertive and that they therefore fail their patients.

[T]o a certain point, you have to stand up for your patient care, because that is at the end of the day your profession. That is what you want to do. Because your patient comes first, irrespective whether the military tells us that rank comes first or a soldier comes first, because that is what they tell us. They tell us you are a soldier first and then your profession. I have heard that many times in my lifetime, soldier first before profession. But if we don't teach them assertiveness, if we don't teach them that, you know, you have to be the advocate for your patient. (Participant FGL2-3)

The issue of rank and how patients with higher ranks are treated or expect to be treated also elicited a number of examples from participants. The allocation of higherranking patients to private rooms with better furniture, equipment and food was one of example of discriminatory treatment based on rank.

But here, especially when we ... they can call from casualty that the colonel or general, whoever is coming. During handover, they will emphasise again [that in] this room and this room there is [a] colonel, there is [a] general, whoever, whoever. This room is a private room, [a] VIP [room] for [a] general, whoever. (Participant FGS1-5)

When I was doing my second stage in 2013 and placed [name withheld], I nursed a patient who was in room 2 (VIP). The first time when I entered the room, I thought I was in one of the expensive hotel rooms! The room had a television, radio, nice couch, beautiful, or with a glass and white cups and fresh flowers. The patient was also eating different food from the other patients, much more appetising! (Participant CIN-4)

Quite a few instances were mentioned of students' experiences involving patients who had expected or demanded preferential treatment on the basis of their rank. In one instance, a patient demanded to be helped first because he had a higher rank than the patient with whom he had been sharing a room. In another instance, a general had demanded to be addressed by his rank and had refused to be treated by students. One participant indicated that an attitude of this nature made it difficult for students 
to perform their duties and that they were wary of entering the rooms of patients with high ranks. The following extract is but one example:

I was in [name withheld], nursing a patient whose rank was a general. According to him, he was supposed to be treated like a general not a patient which makes our work so difficult. He demanded that we address him with his rank not patient. He also did not like it when students nursed him as he was too superior to be nursed by people who did not know what they were doing. This made me sad and confused in a way. Because we are supposed to be learning but how do we learn if we being push aside? We were supposed to be treating a patient not a general. (Participant CIN-6)

One participant had had an experience involving a general who, even as a patient, was still 'commanding' the ward.

Once you go there that general is having that mentality of his owning everyone in the ward even though he is [a] patient and I am [a] nurse. (Participant FGS1-5)

Even patients who were only related to higher-ranking officers had demanded preferential treatment.

[A] general's wife, even if the person is not [in the] military, just because she is married to a general now, she must be recognised. So, most patients ... they all come in, they take the file, first come first serve. So, she came in, she is like, "Can I see doctor so and so?" Then we were like, "Okay, do you have an appointment?" Then the answer was like, "Do you know who I am?" It is like the general's wife or the general cannot sit in the queue; whenever they come, they should just go in that time. So, it is something that is so difficult to deal with. (Participant FGS1-1)

Participants indicated that they found it difficult to nurse patients when they were expected to consider the ranks of the patients before they could treat them.

I feel that in the military, as a nursing student or as a nurse, how it has affected my profession is that you have to be a soldier and a nurse at the same time, especially when we are working in the ... like in [name withheld]. You find people of higher ranks. There you have to consider their ranks before you can treat them. (Participant FGS1-1) 


\section{Discussion}

From the findings, it is evident that the military hierarchical environment, the ranking system and the exploitation of power were considered to be the main challenges in terms of ethical competence confronting students during training.

According to the International Council of Nurses' Code of Ethics ${ }^{23}$ an integral part of nursing is the respect for human rights, inclusive of cultural rights, the right to life and choice, to dignity and to be treated with respect. The Code further stipulates that nursing care should be respectful of and unrestricted by considerations of age, colour, creed, culture, disability or illness, gender, sexual orientation, nationality, politics, race or social status. Of the four principal elements described in the Code that outline the standards of ethical conduct, the first element pertaining to nurses and people clearly delineates the ethical responsibilities of nurses towards patients. It states that:

- in providing care, the nurse should promote an environment in which the human rights, values, customs and spiritual beliefs of the individual, family and community are respected;

- the nurse should ensure that the individual receives accurate, sufficient and timely information in a culturally appropriate manner on which to base consent for care and related treatment;

- the nurse should hold in confidence personal information and use judgement in sharing this information;

- the nurse should share with society the responsibility for initiating and supporting action to meet the health and social needs of the public, in particular those of vulnerable populations;

- the nurse should advocate for equity and social justice in resource allocation, access to health care and other social and economic services; and

- the nurse should demonstrate professional values, such as respectfulness, responsiveness, compassion, trustworthiness and integrity.

From the findings, it became evident that rankism influences students' ability to make principle-based ethical decisions. Firstly, the findings revealed that the military environment is not conducive to upholding respect for human rights. Rankism have also been shown to overrule patients' rights to privacy and confidentially. In terms of meeting the health and social needs of especially vulnerable populations, rankism not only exposes students to exploitation, but also makes it extremely difficult for them to protect vulnerable groups or people with diminished authority, in this case, patients with lower ranks. Rankism further makes students reluctant to ask questions or to speak up when they are uncertain or disagree, which compromises their ability to be assertive, and in turn, their ability to act as advocates for their patients. Furthermore, rankism had a serious effect on the principle of justice. Numerous examples were 
mentioned where patients had to be treated, or where they expected to be treated preferentially based on their ranks. This implies unfair and prejudiced treatment of patients with lower ranks. Lastly, the exploitation of power, concomitant with rankism, also infringes students' dignity, especially in the case where higher-ranking patients refuse to be treated by students.

Numminen et al. refer to an ethical climate as a shared perception of what ethically acceptable behaviour within an organisation is, and how ethical issues should be addressed. ${ }^{24}$ Ethical climate may thus have a positive or negative influence on the development of nurses' ethical and professional competence. ${ }^{25}$ As stated earlier, as a result of rankism, the military environment does not seem to be conducive to the development of students' ethical competence. The ethical climate created by the formal military culture, such as the structure and policies, as well as by the informal culture demonstrated by the behavioural norms, role models and rituals, is affecting students' willingness to show moral courage in issues that have ethical implications.

The role of nursing education and nurse educators in the development of students' ethical competence cannot be disputed. Borhani, Alhani, Mohammadi and Abbaszadeh draw a direct parallel between the contribution of nurse educators, as meta-cognitive guides in teaching ethics, and the ethical reasoning and decisionmaking capability of students. ${ }^{26}$ In a study by Cannaerts, Gastmans and De Casterlé, students perceived presentations on ethics as dull and not motivating, but nonetheless, students and educators underlined the need for presentations on basic ethical principles, as students require a certain amount of ethical knowledge before they can apply it in practice. ${ }^{27}$ Borhani et al. concur that ethics education increases ethical awareness in students, but warns that merely teaching the ethical principles of justice, beneficence, non-maleficence and autonomy will not prepare students when faced with the organisational realities of nursing. ${ }^{28}$ The nature of ethical issues assumes different dimensions in various socio-cultural contexts and the teaching of ethical competency can therefore not be context-free.

\section{Recommendations}

The ranking system in the military will not disappear. In the discussion, the complexity of ethical issues in a military practice environment highlights the importance of ethics education. It is thus recommended that nurse educators adapt their teaching strategies to enhance the ethical competence of students. Case studies or ethical case analysis could be considered a meaningful and appropriate way to provide students with a better understanding of ethical conflicts. Another approach involves the use of patient cases from the students' own clinical practice in group discussions, where, through student participation and interaction, ethical dilemmas from the place of learning are discussed. Furthermore, the use of scenario instruction and the social exchange of ideas with peers and educators could contribute to the development of critical analysis and problem-solving skills. A last teaching approach involves reflective diary sessions where students are encouraged to share their experiences through storytelling. 
It is also important to consider the influence of professional nurses and other healthcare professionals as role models for students in terms of their ways in dealing with the adverse effect of rankism on ethical competence. As such, these role players should be made aware of rankism as part of the hidden curriculum in a military teaching and learning environment and its effect on students' ethical competence so that they can understand how their contribution could improve the outcome of the professional socialisation process of students.

\section{Conclusion}

The study reported here found that, according to students from a military nursing college, the issue of ranks and the subsequent abuse of power in the military environment are factors causing ethical conflict and inhibits ethical decision-making. The accounts of the students point towards a transgression of ethical principles, including the violation of respect, patients' rights to privacy and confidentially, the protection of vulnerable groups, the ability to act as advocates for their patients and fair and equal treatment. The respective studies by Cannaerts et al. and Borhani et al. emphasise the importance of context-specific ethics teaching. ${ }^{29,30}$ It is thus vital that educators of a military nursing college consider the implicit influence of rankism on the development of students' ethical competence and adapt their teaching strategies accordingly.

\section{Disclosure statement}

No potential conflict of interest was reported by the authors.

\section{Endnotes}

${ }^{1}$ M Rassin. "Nurses' professional and personal values". Nursing Ethics 15/5. 2008. 614-630.

${ }^{2}$ FR Jiménez-López, GR Jesus, VS Guillermo \& P Juan. "Values in nursing students and professionals: An exploratory comparative study". Nursing Ethics 23/1. 2016. 79-91.

${ }^{3}$ KK Chitty \& BP Black. Professional nursing: Concepts and challenges (6th ed). Maryland Heights, MO: Saunders Elsevier, 2011.

${ }^{4}$ AW Chuang, FS Nuthalapaty, PM Casey, JM Kaczmarczyk, AJ Cullimore, JL Dalrymple \& L Dugoff. "To the point: Reviews in medical education taking control of the hidden curriculum". American Journal of Obstetrics and Gynaecology 203/4. 2010. 316.e1-316.e6.

${ }^{5}$ EH Gaufberg, M Batalden, R Sands \& SK Bell. "The hidden curriculum: What can we learn from third-year medical student narrative reflections?" Academic Medicine 85/11. 2010. 1709-1716.

${ }^{6} \mathrm{Z}$ Karimi, T Ashktorab, E Mohammadi \& H Abedi. "Influential factors on learning through the hidden curriculum in the perspective of undergraduate baccalaureate nursing students". Journal of Advances in Medical Education \& Professionalism 2/2. 2014. 53. 
${ }^{7}$ FW Hafferty \& JF O'Donnell. The hidden curriculum in health professional education. Dartmouth: Dartmouth College Press, 2015.

${ }^{8} \mathrm{O}$ Numminen, H Leino-Kilpi, H Isoaho \& R Meretoja. "Ethical climate and nurse competence: Newly graduated nurses' perceptions". Nursing Ethics 22/8. 2015. 845-859.

${ }^{9}$ Hafferty \& O’Donnell op. cit.

${ }^{10} \mathrm{~K}$ Zägenhagen. "The influence of the hidden curriculum on professional socialisation of student nurses in a military nursing context". D Litt et Phil thesis. University of South Africa, 2016.

${ }^{11}$ Cambridge Dictionary. Sv "rank". 2017. <https://dictionary.cambridge.org/ dictionary/english/rank> Accessed on 2 November 2017.

${ }^{12}$ Collins English Dictionary. Sv "rankism”. 2017. < $\underline{\text { https://www.collinsdictionary. }}$ com/dictionary/english/rankism $>$ Accessed on 2 November 2017.

${ }^{13}$ MA Staal \& RE King. "Managing a multiple relationship environment: The ethics of military psychology". Professional Psychology: Research and Practice 31/6. 2000. 698.

${ }^{14} \mathrm{~N}$ Jans. "The professionalism of small things: The institutional culture of the Australian Army". In F Vreÿ, AJ Esterhuyse \& T Mandrup (eds), On military culture: Theory, practice and African armed forces. Cape Town: UCT Press, 2015.

${ }^{15}$ Ibid.

${ }^{16}$ Ibid.

${ }^{17}$ N Cannaerts, C Gastmans \& BD de Casterlé. "Contribution of ethics education to the ethical competence of nursing students: Educators' and students' perceptions". Nursing Ethics 21/8. 2014. 861-878.

${ }^{18}$ VD Lachman, JS Murray, K Iseminger \& KN Ganske. "Doing the right thing: Pathways to moral courage". American Nurse Today 7/5. 2012. 24-29.

${ }^{19}$ RW Fuller. Somebodies and nobodies: Overcoming the abuse of rank (no. 132). Gabriola Island: New Society, 2004; C Clarke. "Student perspectives on faculty incivility in nursing education: An application of the concept of rankism". Nursing Outlook 56/1. 2008. 4-8; MR Bleich. "Rankism in nursing and health care". The Journal of Continuing Education in Nursing 45/3. 2014. 108-109; LS Murphy, GA Zangaro \& E Gadsden. "Students' experiences with military nurses as faculty". Journal of Nursing Education 51/4. 2012. 204-211.

${ }^{20} \mathrm{~K}$ Charmaz. Constructing grounded theory. London: Sage, 2014.

${ }^{21}$ DF Polit \& CT Beck. Essentials of nursing research: Appraising evidence for nursing practice (8th ed). Philadelphia: Lippincott Williams \& Wilkins, 2010.

${ }^{22}$ Global Perspectives Consulting. 2013. Enculturation and acculturation. <http:// www.gpccolorado.com/enculturation-acculturation $>$ Accessed on 2 November 2017.

${ }^{23}$ International Council of Nurses. Code of Ethics for Nurses. Geneva, 2012. $<$ http:// www.icn.ch/sites/default/files/inline-files/2012ICNCodeofethicsfornurses \%20eng.pdf $>$ Accessed on 2 November 2017. 
${ }^{24}$ Numminen et al. op. cit.

25 Ibid.

${ }^{26}$ F Borhani, F Alhani, E Mohammadi \& A Abbaszadeh. "Professional ethical competence in nursing: The role of nursing instructors". Journal of Medical Ethics and History of Medicine 3. 2010. 3.

${ }^{27}$ Cannaerts et al. op. cit.

${ }^{28}$ Borhani et al. op. cit.

${ }^{29}$ Cannaerts et al. op. cit.

${ }^{30}$ Borhani et al. op. cit. 